\title{
Orientaciones de metas y clima motivacionales de los otros significativos en jóvenes de jugadores extremeños de balonmano Orientations and motivational climates of the other significatives in younghandballplayersfromExtremadura
}

\author{
*Pedro Antonio Sánchez Miguel, *Francisco Miguel Leo Marcos, *Francisco Rafael Gómez Corrales \\ *David Sánchez Oliva, **Ernesto de la Cruz Sánchez y *Tomás García Calvo \\ *Universidad de Extremadura (España), **Universidad Murcia (España)
}

\begin{abstract}
Resumen: La motivación es un constructo empleado para explicar los aumentos de participación deportiva en iniciación. Por ello, el objetivo del presente estudio es analizar las orientaciones de meta de jóvenes jugadores de balonmano, así como los climas motivacionales de los compañeros, padres y entrenadores. La muestra está formada por 127 jugadores de balonmano con edades comprendidas entre los 11 y los 16 años, pertenecientes a equipos federados de la comunidad autónoma de Extremadura. Los resultados nos muestran la asociación de los constructos de la teoría de metas entre los diferentes significativos analizados. Además, el análisis de regresión demuestra la importancia que tienen los padres en la conformación de las orientaciones de los jugadores de balonmano. Finalmente, se discuten los resultados analizados y se extraen unas conclusiones en la línea de aumentar el conocimiento de las orientaciones y climas motivacionales en jóvenes jugadores de balonmano.
\end{abstract}

Palabra clave: Balonmano, iniciación deportiva, motivación.

\begin{abstract}
Motivation is a construct used to explain the increase of sport participation in the initiation context. For it, the aim of this work is to measure young handball players' orientation, as well as motivational climates of peers, parents and coaches. The sample is formed by 127 handball players, which ages ranging from 11 and 16 years old, belonged to different affiliated teams of the Extremadura region. The results showed the association among the different significatives analyzed in the goal achievement theory constructs. Besides, regression analyses showed the importance of the parents in the formation of the handball player's orientations. Finally, the outcomes are discussed and we take some conclusions out in the light of enhance the knowledge of young handball players orientations and motivational climates.
\end{abstract}

Key words: Handball, sport initiation, motivation.

\section{Introducción}

En España, está aumentando continuamente el interés de todos los sectores de la población por la práctica físico deportiva (García Ferrando, 2006). En este sentido, se ha demostrado que la motivación es una de las variables psicológicas más estudiadas para conocer dichos aumentos de interés por la participación deportiva. Esta variable representa las fuerzas internas y/o externas que producen la iniciación, dirección, intensidad y persistencia de comportamiento (Vallerand y Thill, 1993). Cashmore (2002) indica que la motivación es un estado o proceso interno que activa, dirige y mantiene la conducta hacia un objetivo. En este sentido, varios autores han encontrado que tanto la intensidad como la dirección que adoptan los motivos de práctica son importantes para la continuidad en una actividad físico - deportiva (Robison y Carron, 1982; Cervellóy cols., 2007; Ntoumanis, 2005; Ryska, Hohensee, Cooley y Jones, 2002).

En esta línea, la teoría de metas de logro (Nicholls, 1989; Ames, 1992) ha sido una de las teorías motivacionales que ha producido la mayor cantidad de investigaciones en el campo del deporte y ejercicio psicológico (ver para revisión, Duda, 2001). Esta teoría explica que existen dos tipos de orientación motivacional en función del concepto de habilidad percibida. El primero es la orientación hacia el ego, consistente en valorar la habilidad comparándose con otras personas, dando más importancia al resultado de nuestro comportamiento que al esfuerzo y a la ejecución(Duda, 2005). Por el contrario, la orientación a la tarea se caracteriza por valorar la habilidad de forma criterial, es decir, en función de uno mismo, así como por dar mucha importancia al esfuerzo y a la ejecución por encima de los resultados (Ames, 1992). Los individuos no se comparan con ningún elemento externo, y se ven ellos mismos como capaces para aprender algo nuevo y progresar en el

Fecha recepción: 05-01-09 - Fecha envío revisores: 10-01-09 - Fecha de aceptación: 20-04-04 Correspondencia: Pedro Antonio Sánchez Miguel

Facultad de Ciencias del Deporte. Universidad de Extremadura.

C/ Avenida de la Universidad s/n. C.P.:10071. Cáceres. España.

E-mail: pedroantonio.sanchez@juntaextreamdura.net aprendizaje de la destreza. Una orientación hacia la tarea, está asociada con patrones motivacionales, afectivos y comportamentales más positivos que una orientación hacia el ego. Así, los individuos orientados hacia la tarea tienden a poner más esfuerzo es los deportes, demostrando mayor persistencia, mayor divertimiento y expresando mayor satisfacción cuando practican deportes. Por contra, individuos orientados al ego demuestran menos patrones deportivos motivacionales caracterizados por demostrar menos esfuerzo en practicar deportes, menor divertimiento, y dejando la actividad cuando ellos encuentran pequeñas dificultades o se ven ellos mismos como poco habilidosos (Castillo y cols., 2002; Cervelló y cols., 1999; Cervelló y Santos-Rosa, 2001).

Nicholls (1984) sugiere que el modo en que los sujetos construyen su concepto de lo que es capacidad, varía en función del desarrollo y diferencias individuales que están influidas por aspectos disposicionales y de situación. Por ello, las bases donde se asientan las predicciones cognitivas, afectivas y conductuales se encuentran en la forma en que los sujetos interpretan la competencia o habilidad percibida, es decir, desde una orientación a la tarea y al ego, y en el nivel de esa competencia.

De esta manera, son muchos los estudios que han analizado las orientaciones de meta con la percepción de capacidad en el ámbito deportivo, tanto autorreferencial (la propia capacidad para practicar deporte) como normativa (comparándose con los demás). Duda y Nicholls (1992) demostraron que tanto la orientación a la tarea como la orientación al ego se asociaron positivamente a la percepción de capacidad normativa. Cervelló (1999) señala que las personas que están orientadas al ego tienen dificultades para mantener el sentimiento de competencia en la actividad, presentan patrones de conducta inadaptada y abandonan la actividad cuando su percepción de competencia es baja. Además, este autor indica que las personas orientadas hacia la tarea se esfuerzan en la actividad y persisten en la práctica independientemente de la percepción de competencia y el fracaso se percibe como un elemento del aprendizaje que ayuda a mejorar. Walling y cols. (2002) demostraron que los sujetos con una alta orientación a la tarea tendían a mostrar mayor diversión e interés con la práctica deportiva, independientemente de su nivel de competencia o de su resultado en la competición. 
La teoría de las metas de logro establece que en función de las características que perciba el sujeto en el contexto en el que se encuentre, se puede diferenciar entre un clima motivacional implicante hacia el ego o hacia la tarea (Ames, 1992). Así, los individuos se socializan hacia diferentes metas de logro, mientras los otros significativos (padres, entrenadores, compañeros, etc.) crean un clima motivacional o conjunto de señales implícitas o explícitas percibidas en el entorno, a través de las cuales se definen las claves del éxito y el fracaso, siendo denominado por Ames (1992) y Nicholls (1989) como clima competitivo y clima de maestría, mientras que otros autores, lo denominan clima motivacional implicante al ego y clima motivacional implicante a la tarea (Cervelló y Santos-Rosa, 2001; Escartí y cols., 1999) o clima motivacional orientado al rendimiento y clima motivacional orientado al aprendizaje (Papaioannou,1995). Ntoumanis y Biddle(1999) destacan que el clima motivacional es un aspecto importantísimo en la explicación de de aspectos como el esfuerzo, persistencia, comportamientos adaptativos o desadaptativos, cogniciones... en el contexto de la actividad física.

Los otros significativos que componen el clima motivacional hacen referencia al modo en el que se utilizan los sistemas de recompensas, la manera en la que se diseñan las prácticas, la forma en la que se agrupan a los sujetos y la manera en la que las figuras de autoridad evalúan el rendimiento (Escartí y Gutiérrez, 2006). Estos significativos (entrenadores, padres o compañeros) son un pilar fundamental en la explicación del comportamiento del joven deportista en el contexto deportivo.

De esta manera, el clima orientado a la tarea se encuentra asociado positivamente con las metas orientadas a la tarea, diversión, satisfacción, interés y motivación intrínseca, aprendizaje cooperativo, mejora personal, aumento de la participación, mayor aprendizaje, compromiso deportivo... (Cecchini y cols., 2001; Ntoumanis, 2002; Sousa y cols., 2007). Por el contrario, el clima orientado al ego, se encuentra relacionado positivamente con la orientación al ego, afectividad negativa y sentimientos de presión, se demuestra promovedor de la competición interpersonal mediante el uso de la comparación normativa y pública (Cecchini y cols., 2001; Ntoumanis, 2002).

La relación entre climas motivacionales es confusa. Algunos autores como Biddle y cols. (1995) y Papaioannou (1994) defienden que los climas motivacionales son independientes, mientras que otros como Kavussannu y Roberts (1996) opinan que los climas se encuentran negativamente relacionados. Gano-Overway y Ewing (2004), analizaron la influencia de las orientaciones sobre los climas. Así, con 162 adolescentes participantes en un programa de actividad física a lo largo de cuatro meses demostraron las relaciones entre el clima percibido y las orientaciones motivacionales, correlacionando el clima ego con la orientación al ego y el clima tarea con la orientación tarea. Igualmente, descubrieron que la percepción de un determinado tipo de clima puede modificar a largo plazo la orientación motivacional, sobre todo cuando el clima percibido es diferente a la principal orientación motivacional.

Asimismo, los climas motivacionales generados por los otros significativos actúan de diferente forma según la edad de los sujetos. Así, en un principio parece ser que los padres tienen una mayor influencia en la participación de los niños en la práctica deportiva (Duda y cols., 2005). En este sentido, destacamos el estudio de White (1998), que contemplaron la importancia del clima percibido procedente de los padres. Así, con una muestra de 279 adolescentes de diferentes deportes, hallaron que aquellos con alta orientación a la tarea y baja orientación al ego, percibían un clima implicante a la tarea, mientras que aquellos con alta orientaciónal ego y baja orientación a la tarea percibían un climaimplicante al ego. En España, Ruiz y García (2003) demostraron que la familia posee una influencia positiva en la configuración de los hábitos deportivos, principalmente en las primeras edades del joven deportista.

A partir de la adolescencia, parece que la influencia de la familia comienza a declinar y el grupo de pares se vuelve muy importante, especialmente los iguales del mismo género, proporcionando apoyo y reconocimiento social (Castillo y cols., 2004).

El entrenador también cumple un papel destacado en el proceso de socialización deportiva, debido a la proximidad para juzgar y proporcionar recompensas o castigos a los deportistas y debido a la influencia que éste ejerce como líder del grupo deportivo (Giwin, 2001). Su papel se ha estudiado generalmente en poblaciones de deportistas jóvenes. Además, como señala López Martínez (2006) este significativo es un medio de transmisión de valores y juega un rol como ejemplo para los jugadores. Asimismo, se ha demostrado que la actitud del entrenador es esencial a la hora de provocar en el joven curiosidades que hagan que aumenten la motivación y el interés por la práctica de forma continua (Ruiz-Risueño y De la Cruz, 2008).

En Extremadura, la iniciación deportiva en está alcanzando unos niveles de participación deportiva muy elevados. En este sentido, el programa Judex (Juegos Deportivos Extremeños) presente año tras año más participación en el contexto del deporte escolar. Este programa fomenta la práctica deportiva en centros educativos y entidades deportivas de la Comunidad Autónoma de Extremadura, desarrollándose la convivencia deportiva a través de jornadas y encuentros de manera regulary formativa.

Por todo ello, el objetivo del presente estudio es analizar las orientaciones de meta disposicionales de participantes en balonmano y los climas motivacionales de padres, entrenadores y compañeros, además de conocer la asociación entre los componentes de la teoría de las metas de logro de esos deportistas en el contexto de la iniciación deportiva en Extremadura.

\section{Método}

\subsection{Muestra}

La muestra de la investigación estaba compuesta por 127 jugadores de balonmano. Los participantes eran de género masculino $(n=75)$ y femenino ( $\mathrm{n}=52$ ), y con edades comprendidas entre los 11 y los 16 años $(M=12.37 ; D T=1.48)$. Todos los jugadores que formaban parte de la muestra, pertenecían a equipos federados que jugaban en competiciones organizadas en categoría alevín ( $n=49)$, infantil $(n=50)$ y cadete $(n=22)$, teniendo cada participante una ficha federativa con sus datos personales y deportivos.

\subsection{Instrumentos}

Orientaciones motivacionales. Para medir las orientaciones de metas disposicionales se utilizó una versión reducida del Perception of Success Questionnaire (POSQ: Roberts y cols., 1998). Consta de un total de 12 ítems agrupados en dos factores principales, como son la orientación al ego (6ítems, ej.: Realizo las tareas mejor que otros jugadores) y la orientación a la tarea (6ítems, ej.: Consigo hacer alguna cosa que antes no hacía).

Clima motivacional percibido en los iguales. Se empleó la versión validada al castellano del Peer Motivational Climate in youth sport Questionaire (PEERMCYSQ: Ntoumanis y Vazou, 2005). Este instrumento consta de cuatro factores, como son apoyo, autonomía, competencia y relaciones. Se eligieron los dos que mostraban mayor peso factorial, el primer fue el factor Apoyo, denominado como clima implicante a la tarea (ej.: En mi grupo de entrenamiento, los compañeros dan consejos a los compañeros para ayudarles a progresar) y el segundo factor fue la Competencia, denominado como clima implicante al ego (ej.: En mi grupo de entrenamiento, los compañeros intentan hacerlo mejor que los demás).

Clima motivacional de los entrenadores. Se utilizóla adaptación al castellano del Perceived Motivational Climate in Sport Questionnaire (PMSCQ - 2: Newton y cols., 2000). Nos hemos centrado en los factores de segundo orden, como son el clima que implica al rendimiento o ego (ej.: El entrenador motiva a los jugadores cuando juegan mejor que sus compañeros) y el clima que implica al aprendizaje o tarea (ej.: El entrenador favorece que los jugadores se ayuden unos a otros en los entrenamientos y partidos).

Participación de los padres en la práctica deportiva o Clima motivacional de los padres. Para su medición se ha utilizado la versión adaptada al castellano del Parental Involvement Sport Questionaire 
(PISQ: Lee y Mclean, 1997). Dicho cuestionario cuenta con un total de 20 ítems que se agrupan en cuatro factores principales: implicación activa (5 ítems, ej.: Tus padres tienen un papel activo en el funcionamiento del club), apoyo y compresión (6 ítems, ej.: Tus padres te elogian por lo que has hecho bien, aunque el partido se haya perdido), comportamiento directivo ( 5 ítems, ej.: Tus padres antes de los partidos, te dicen cómo has de jugar) y presión (4 ítems, ej.: Tus padres te presionan para que entrenes mejor). Los dos primeros factores hacen referencia a los comportamientos adecuados o clima motivacional implicante a la tarea de los padres, y los dos último factores a los comportamientos inadecuados o la clima motivacional implicante al ego.

\subsection{Procedimiento}

Para llevar a cabo la recogida de datos, desarrollamos un protocolo de actuación para que la obtención de datos fuera similar en todos los participantes implicados en la investigación.

En primer lugar, contactamos con los entrenadores y monitores de diferentes equipos que podrían formar el conjunto de participantes de la investigación. A través de una nota informativa, se puso al corriente a los padres de dicha investigación, de sus objetivos y de la utilización de los datos obtenidos, rogándoles que la devolvieran firmada si aceptaban que sus hijos participaran en la investigación.

También se informó a los deportistas de que su participación era voluntaria y las respuestas serían tratadas confidencialmente. Los participantes (jugadores y entrenador) rellenaron los cuestionarios en el vestuario, sin la presencia del entrenador, de manera individual y en un clima que les permitía concentrarse sin tener ningún tipo de distacción.

El investigador principal estuvo presente en el momento en que los sujetos completaban los cuestionarios, e insistió en la posibilidad de preguntar cualquier tipo de duda que apareciese durante el proceso. Asimismo, se entregó el cuestionario a cada participante para que lo devolviera al día siguiente rellenado por su padre o madre.

\subsection{Análisis de los datos}

El análisis de resultados se ha realizado mediante la utilización del programa estadístico SPSS 15.0. En primer lugar, se eliminaron todos aquellos participantes que dejaban total o gran parte de los cuestionarios sin responder, así como aquellos que respondieron al cuestionario de forma aleatoria o al azar. El porcentaje de muestra eliminado no fue superior al 2\%. Las técnicas de análisis estadístico utilizadas han sido: el análisis de fiabilidad, el análisis descriptivo, el análisis correlacional y el análisis de regresión lineal.

Tabla 1. Estadísticos descriptivos

\begin{tabular}{lrl} 
& $M$ & $D T$ \\
\hline Orientación Tarea & 2.96 & 1.08 \\
Orientación Ego & 4.38 & .56 \\
Clima Tarea Compañeros & 4.26 & .62 \\
Clima Ego Compañeros & 2.11 & .91 \\
Clima Tarea Entrenador & 3.68 & .64 \\
Clima Ego Entrenador & 3.22 & 1.09 \\
Implicación Club Padres & 3.15 & .98 \\
Apoyo Padres & 4.01 & .90 \\
Comportamiento Directivo Padres & 2.06 & 1.04 \\
Presión Padres & 2.99 & 1.07 \\
\hline
\end{tabular}

\section{Resultados}

En la tabla 1 se presentan los estadísticos descriptivos de las variables analizadas. En ella, se puede observar los altos valores que presentan los jóvenes jugadores de balonmano en la orientación a la tarea $(M=$ 4.38), en contraposición a los moderadamente bajos niveles de orientación al ego que manifiestan $(M=2.96)$. Asimismo, se puede destacar cómo el clima tarea creado por los compañeros $(M=4.26)$ y el entrenador $(M=3.68)$, además de la implicación al club de los padres $(M=$ 3.15) y su apoyo $(M=4.01)$ presentan valores por encima de hallados en el clima ego de compañeros $(M=2.11)$ y entrenador $(M=3.22)$, así como los comportamientos directivos $(M=2.06)$ de los padres y la presión $(M=2.99)$ de éstos hacia sus hijos e hijas.

En cuanto al análisis de fiabilidad, todos los factores poseen puntuaciones por encima o cercanos a .70 en el coeficiente de Alpha de Cronbach, por lo que la fiabilidad de los instrumentos resulta aceptable.

En la tabla 2, se muestran las correlaciones entre las orientaciones motivacionales de los participantes, los climas generados por compañeros y entrenador y la implicación de los padres en la práctica deportiva de sus hijo/as. En relación con el clima tarea generado por los compañeros destacamos la asociación positiva $(\mathrm{p}<$.01) con el clima tarea del entrenador (.387) y con la implicación al club de los padres (.365).

Asimismo, esta variable mantiene una asociación negativa ( $\mathrm{p}<.01)$ con el clima ego generado por los compañeros (-.344). En cuanto al clima tarea generado por el entrenador, resaltamos la relación significativa y Tabla 2. Correlaciones entre los factores del estudio

\begin{tabular}{|c|c|c|c|c|c|c|c|c|c|}
\hline \# & 1 & 2 & 3 & 4 & 5 & 6 & 7 & 8 & 9 \\
\hline \multicolumn{10}{|l|}{ 1. Orientación Tarea } \\
\hline \multicolumn{10}{|l|}{ 2. Orientación Ego } \\
\hline \multicolumn{10}{|l|}{ 3. Clima Tarea } \\
\hline Compañeros & $.425(* *)$ & -.078 & & & & & & & \\
\hline \multicolumn{10}{|l|}{ 4. Clima Ego Compañeros - } \\
\hline \multicolumn{10}{|l|}{ 5. Clima Tarea Entrenador . } \\
\hline 6. Clima Ego Entrenador & .132 & $.449(* *)$ & $.044=1$ & $.371(* *)$ & -.071 & - & & & \\
\hline 7. Implicación Club Padres & .114 & .15 & .117 & $.189(*)$ & .164 & $.217(*)$ & - & & \\
\hline 8. Apoyo Padres & $.343(* *)$ & -.066 & $.365(* *)$ & .047 & $.356(* *)$ & $.190(*)$ & $.586(* *)$ & - & \\
\hline 9. Comp. Directivo Padres & -.098 & $.274(* *)$ & -.181 & $.557(* *)$ & .127 & $.326(* *)$ & $.485(* *)$ & $.207(*)$ & - \\
\hline 10.Presión Padres & .083 & .127 & .123 & $.246(* *)$ & .113 & .134 & $.310(* *)$ & $.277(* *)$ & $.297(* *)$ \\
\hline
\end{tabular}

positiva $(\mathrm{p}<.01)$ que mantiene con los apoyos de los padres (.356). Es decir, los padres apoyan más la práctica deportiva de sus hijo/as cuanto mayor sea la capacidad del entrenador de no utilizar un criterio normativo en su evaluación, basar el progreso únicamente en el resultado, realizar comparaciones entre compañeros, etc.

En este sentido, destacamos que el clima ego generado por el entrenador tiene una relación positiva $(p<0.01)$ con la orientación al ego (.449) de los participantes, el clima ego generado por los compañeros (.371) y el comportamiento directivo de los padres (.326), lo que nos reafirma lo expresado anteriormente.

En la tabla 3 se muestra el análisis de regresión utilizando como variable dependiente la orientación a la tarea de los participantes. En el primer paso se obtuvo como variable predictora el apoyo de los padres. Es variable tiene una capacidad predictiva del $17 \%$ de varianza. En el segundo paso, que explica un 5\% de la varianza, aparece el clima tarea del entrenador. Se puede destacar que esta variable tienen un peso de predicción bajo, ya que posee un valor de predicción menor al 10\% de varianza explicada.

Por otra parte, en la tabla 4 se muestra el análisis de regresión utilizando como variable dependiente la orientación al ego de los jugadores de balonmano. En el primer paso, observamos cómo el clima implicante al ego de los compañeros en la variable con mayor peso de predicción (16\%). Por tanto, a mayor clima motivacional hacia el ego promovido por los compañeros, mayor será la orientación motivacional 


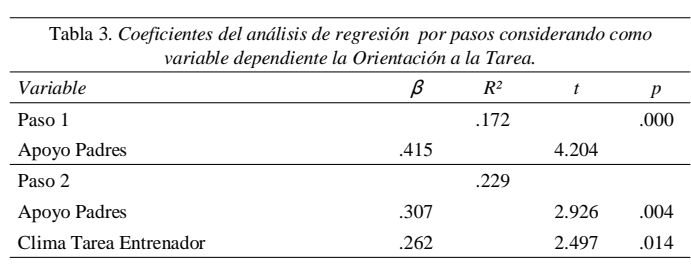

\begin{tabular}{lcccc}
\hline \multicolumn{5}{c}{ Tabla 4. Coeficientes del análisis de regresión por pasos considerando como } \\
variable dependiente la Orientación al Ego.
\end{tabular}

hacia el ego. En un segundo paso, aparecela presión de los padres como variable que explica un $6 \%$ de la varianza. El resto de variables quedan excluidas porque tienen un valor de predicción menor al 10\% de la varianza explicada.

\section{Discusión}

Tras el análisis de los resultados, podemos indicar que los jugadores de balonmano analizados presentan unos niveles de orientación y climas motivacionales implicante a la tarea superior a los niveles implicantes al ego. Estos resultados son consistentes con los encontrados en otros estudios que analizan los aspectos motivacionales en diferentes contextos como el deporte olímpico y paralímpico (Pensgaard y cols., 1999), recreativo (Cervelló y Santos-Rosa, 2001) y educacional (Cervelló y Santos-Rosa, 2000; Flores y cols., 2008). Asimismo, hemos observado la asociación existente entre los componentes la Teoría de las Metas de Logro(Nicholls, 1989). Concretamente destacamos cómo se relacionan los factores ego en el clima de los compañeros y entrenadores, además de la orientación de los participantes. Igualmente, los constructos del factor Tarea también se asocian entre los significativos analizados. En este sentido, este hallazgo de asociación de los constructos de la misma perspectiva de meta se ha encontrado en anteriores estudios (Treasure y cols., 2001; Cervelló y Santos-Rosa, 2001; Vazou y cols., 2006), siendo justificado por estos autores a partir de la importancia que adquiere la Orientación disposicional en la percepción de las estrategias y dimensiones que se asocian a un determinado clima motivacional (Duda, 2005). De esta manera, un sujeto que tenga una elevada Orientación a la Tarea, tenderá a percibir de forma más acentuada las claves motivacionales del clima que impliquen hacia la Tarea, sucediendo lo mismo en el constructo Ego. Así, se demuestra que las estructuras y demandas del ambiente de aprendizaje influyen en la adopción de una u otra implicación por parte de los jugadores, así como sus patrones motivacionales resultantes (Cervelló y Santos-Rosa, 2000).

Además, destacamos la fuerte asociación existente entre la implicación al club de los padres y el apoyo de éstos con los constructos tarea. Por el contrario, la presión de los progenitores y su comportamiento directivo se ha asociado con el factor ego. Estos resultados han sido confirmados por varios estudios como el de Eccles y Harold (1991), que comentan que durante los años de formación la influencia de los padres se percibe como un elemento revelador en la motivación de logro de los niños. Asimismo, Carr y cols. $(1999,2000)$ y Carr y Weigand (2002) corroboraron esta afirmación, considerando que la influencia de padres, profesores, compañeros y héroes deportivos sobre los patrones de logro de niños en el deporte pueden estar relacionados con las orientaciones de metas de los chicos. White (1996) encontraron que las orientaciones de metas de chicas adolescentes estaban relacionadas con el clima motivacional generado por los padres. Así, las percepciones que las participantes tenían de que sus padres enfatizasen el aprendizaje y el divertimento, estaba asociado con una orientación a la tarea, mientras que la percepción de enfatizar el éxito sin esfuerzo se relacionaba con la orientación al ego. Esta afirmación fue corroborada más tarde por un estudio de Kavussanu y Guest (1998) en el que demostraron la asociación entre la orientación a la tarea y un clima implicante a la tarea de los progenitores, tanto padres como madres. Anteriormente, Duda y Horn (1993) habían analizado las relaciones existentes entre las orientaciones de padres e hijos, demostrando que los chicos que tenían valores altos en orientación a la tarea, percibían a sus padres con grandes niveles de orientación a la tarea, ocurriendo exactamente igual con la orientación alego.

Para tratar de dilucidar la cuestión de la importancia que adquiere cada significativo, podemos observar en el modelo de ecuaciones estructurales. Así, el análisis de regresión efectuado nos indica la importancia de la influencia de los padres sobre las disposiciones motivacionales de los hijo/as, ya que el apoyo de los padres y la presión de éstos hacia sus hijo/as se muestran predictores de la orientación a la tarea y al ego respectivamente. Estos resultados están en la línea de lo encontrado por White (1998) y Bergin y Habusta (2004) quienes demostraron la importancia de los padres en la orientación motivacional de sus hijos. Asimismo, los trabajos de Duda y cols. (2005) y de Jowett y TimsonKatchis (2005) encontraron resultados similares. Sin embargo, y a pesar de la importancia que tienen los padres en la conformación de las disposiciones motivacionales de los hijo/as , el estudio realizado en España por Romero y cols. (2009) demuestra la falta de implicación de los padres en la vida deportiva de sus hijos en general, tanto hacia la competición como hacia el entrenamiento.

En consonancia con los resultados hallados en el análisis de regresión teniendo como variable dependiente la orientación al ego, podemos señalar que la mayor capacidad predictora del clima motivacional implicante al ego de los compañeros puede ser debida a los conflictos que se producen dentro del equipo, falta de apoyo entre ellos, falta de cooperación, excesiva competición entre ellos... (Ntoumanis y Vazou, 2005). Nuestros resultados no son consistentes por los hallados por Escartí y cols. (1999), quienes indicaron que los adultos tenían más importancia en la conformación de los climas implicantes al ego de los participantes que sus compañeros.

En nuestros resultados observamos como los entrenadores manifiestan una capacidad predictora en la orientación en la tarea de los participantes, no presentando ninguna capacidad predictora en la orientación al ego. Estos resultados nos sugieren la influencia positiva que el entrenador genera sobre los deportistas, teniendo en cuenta que éste mantiene una relación muy fuerte con la motivación de los jóvenes en la medida que desarrolla su rol en las instrucciones y valoraciones que realiza (Keegan y cols. 2009). Así, se ha demostrado que un clima implicante a la tarea por parte del entrenador, está asociado con la mayor satisfacción de los participantes, niveles de autoestima, bienestar físico y mental. .. además de estar asociado con el rendimiento en la tarea que se esté realizando (Reinboth y Duda, 2006).

Finalmente, podemos destacar que además de la importancia en la conformación de las orientaciones disposicionales de los padres sobre sus hijos, parece ser que un mayor apoyo de los padres sobre la práctica deportiva de sus hijos, está relacionada con un aumento del rendimiento de éstos (Van Yperen y Duda, 1999).

\section{Conclusiones}

La conclusión más importante del presente estudio radica en la confirmación de la asociación de los constructos de la Teoría de metas entre los diferentes significativos analizados. Además hemos observado a través del análisis de regresión de la importancia que tienen los padres en la conformación de las orientaciones de los jugadores de balonmano. Aunque, tal y como han indicado varios autores (Brustad, 1992; Duda, 2005), la importancia de los otros significativos varía en función de diferentes parámetros como pueden ser la edad de los sujetos, el género y el tipo de deporte y nivel competitivo del mismo, por lo que nuestros resultados deben acotarse a la población que representa nuestra muestra. Finalmente, en la línea con los resultados encontrados parece ser que estos mayores niveles de orientación a la tarea y percepción de clima tarea entre los participantes pueden conducir a una mayor satisfacción 
y bienestar de los participantes (ver para revisión, Duda, 2001) tan importante en contextos como la iniciación deportiva que hemos analizado.

\section{Estrategias de intervención}

Tras la extracción de las conclusiones del presente trabajo, podemos establecer una serie de estrategias para la optimización de la orientación a la tarea de los participantes y su percepción de clima tarea por parte de los otros significativos. Así, tras demostrar la fuerte asociación del apoyo de los padres y su implicación con los constructos tareas, al igual que el clima generado por el entrenador, una estrategia a seguir puede ser las desarrolladas por García Calvo y cols. (2009) dónde realizan charlas coloquios con padres y entrenadores en el contexto de la iniciación deportiva con el objetivo de transmitir los valores positivos de la actividad física y deportiva, y disminuir comportamientos agresivos y antisociales. En estas charlas se implica a los padres en la actividad deportiva de sus hijos y, se indican los efectos negativos que una excesiva presión de ellos sobre sus hijos puede ocasionar en estos últimos. Igualmente, estas charlas pueden generar vínculos de amistad entre los participantes, lo que traducirá en una mayor satisfacción de éstos.

Además, para la mayor implicación al club y apoyo de los padres en la práctica deportiva de sus hijos, se les solicita la firma de «contratos» en el que se comprometan a mantener unos comportamientos adecuados como no criticar y/o gritar a su hijo/a, y apoyar a éstos en cualquier necesidad que presenten a nivel deportivo. Asimismo, la distribución de trípticos que muestren la relación entre los comportamientos de apoyo e implicación sobre los beneficios saludables de sus hijos en la práctica deportiva, puede modificar los comportamientos de apoyo y presión de los progenitores sobre sus hijos.

Con respecto a los entrenadores, se realizan talleres y dinámicas de grupo dónde se presentan las estrategias seguidas por Duda, Ntoumanis, Mahoney, Larson, y Eccles (2005) para conseguir un clima implicante a la tarea en sus entrenamientos.

\section{Bibliografía}

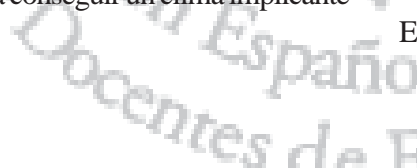

Ames, C. (1992). Classrooms: Goals, structures, and student motivation. Journal of Educational Psychology, 84, 261-271.

Biddle, S. J. H., Cury, F., Goudas, M., Sarrazin, P., Famose, J. P., y Durand, M. (1995). Development of scales to measure perceived physical education class climate: A cross-national project. British Journal of Educational Psychology, 65, 341-358.

Bergin, D.A. y Habusta, S.F. (2004). Goal orientations of young male ice hockey players and their parents. Journal of Genetic Psychology, 165, 383-397.

Brustad, R. J. (1992). Integrating socialization influences into the study of children's motivation in sport. Journal of Sport y Exercise Psychology, 14(1), 59-77.

Carr, S., Weigand, D. A., y Hussey, W. (1999). The relative influence of parents, peers on children and adolescents. Journal of Sport Pedagogy, 5, 28-50.

Carr, S., Weigand, D. A., y Jones, J. (2000). The relative influence of parents, peers and sporting heroes on goal orientations of children and adolescents in sport. Journal of Sport Pedagogy, 6(2), 34-55.

Cashmore, E. (2002). Sport Psychology: the key concepts. Londres: Routledge.

Castillo, I., Balaguer, I. y Duda, J. L. (2002). Las perspectivas de meta de los adolescentes en el contexto deportivo. Psicothema, 14, 280287.

Castillo, I., Balaguer, I., Duda, J. L., y García-Merita, M. L. (2004). Psychosocial factors associated with sports practice during adolescence. Revista Latinoamericana de Psicología, 36(3), 505515.

Cecchini, J. A., González, C., Carmona, A., Arruza, J., Escartí, A. y Balagué, G. (2001). The Influence of the Teacher of Physical Education on Intrinsic Motivation, Self-Confidence, Anxiety, and
Pre- and Post-Competition Mood States. European Journal of Sport Science. 1, 4-22 (2), 160-171.

Cervelló, E. M. (1999) Variables psicológicas relacionadas con la elección de tareas deportivas con diferente nivel de dificultad. Consideraciones para el diseño de programas motivacionales de entrenamiento psicológico en el deporte. Motricidad, 5, 35-52.

Cervelló, E. M., Escartí, A., Guzmán, J. F. (2007). Youth sort dropout from the achievement goal theory. Psicothema, 19, 65-71.

Cervelló, E. M., y Santos-Rosa, F. J. (2001). Motivation in Sport: and achievement goal perspective in young spanish recreational athletes. Perceptual and Motor Skills, 92, 527-534.

Cervelló, E.M. y Santos-Rosa, F.J. (2000). Motivación en las clases de Educación Física: un estudio de la perspectiva de las metas de logro en el contexto educativo. Revista de Psicología del Deporte, 9, 5170.

Duda, J. L. (2001). Goal perspective research in sport in sport: Pushing the boundaries and clarifying some misunderstandings. In G. C. Roberts (Ed.), Advances in motivation in sport and exercise (pp. 129-182). Champaign, IL: Human Kinetics.

Duda, J. L. (2005). Motivation in Sport: The Relevance of Competence and Achievement Goals. In A. J. Elliot y C. S. Dweck (Eds.), Handbook of competence and motivation (pp. 273-308). New York: Guilford Publications.

Duda, J. L., Ntoumanis, N., Mahoney, J. L., Larson, R. W., y Eccles, J. S. (2005). After-school sport for children: Implications of a taskinvolving motivational climate. Mahwah, NJ, US: Lawrence Erlbaum Associates, Publishers.

Duda, J. L., y Nicholls, J.G. (1992). Dimensions of achievement motivation in schoolwork and sport. Journal of Educational Sport Psychology, 84, 110.

Eccles, J. E., y Harold," R. D. (1991). Gender differences in sport involvement:Applying the Eccles expectancy-value model. Journal ofApplied Sport Psychology, 3, 735.

Escartí, A., Roberts, G.C., Cervelló, E.M. y Guzmán, J.F. (1999). Adolescent goal orientations and the perception of criteria of success used by significant others. International Journal of Sport Psychology, 30, 309-324.

Escartí, M. y Gutiérrez, M. (2006). Influencia de padres y profesores sobre las orientaciones de meta de los adolescentes y su motivación intrínseca en educación física. Revista de psicología del deporte, 15 (1), 23-35.

Flores, J., Salguero, A. y Márquez, S. (2008). Goal orientations and perceptions of the motivational climate in physical education classes among Colombian students. Teaching and Teacher education, 24 (6), 1141-1149.

Gano-Overway, L.A., y Ewing, M.E. (2004). Alongitudinal perspective of the relationship between perceived motivational climate, goal orientations, and strategy use. Research Quarterly for Exercise and Sport, 75(3), 315-325.

García Calvo, T., Sánchez Miguel, P.A., Leo, F.M., Sánchez Oliva, D. y Gómez Corrales, F.R. (2009). Escuela del Deporte. Una Campaña para la promoción de los valores del deporte. Consejería de los Jóvenes y el Deporte. Junta de Extremadura.

García Ferrando, M. (2006). Veinticinco años de análisis del comportamiento deportivo de la población española (1980 - 2005). Revista internacional de sociología, 44, 15-38.

Giwin, K. B. (2001). Goal orientations of adolescents, coaches, and parents: Is there a convergence of beliefs? Journal of Early Adolescence, 21(2), 227-247.

Jowett, S., y Timson-Katchis, M. (2005). Social Networks in Sport: Parental Influence on the Coach-Athlete Relationship. Sport Psychologist, 19(3), 267-287.

Kavussanu, M., y Roberts, G C. (1996). Motivation in physical activity contexts: The relationship of perceived motivational climate to intrinsic motivation and self-efficacy. Journal of Sport y Exercise Psychology, 18(3), 264-280. 
Keegan, R.J., Harwood, C.G., Spray, C.M. y Lavallee, D.E. (2009). A qualitative investigation exploring the motivational climate in early career sports participants: Coach, parent and peer influences on sport motivation. Psychology of Sport and Exercise, 10(3), 361372.

Lee, M. J., y McLean, S. (1997). Sources of parental pressure among age group swimmers. European Journal of Physical Education, 2, 167-177.

López Martínez, J. (2006). Educación Física y Deporte Escolar. Retos. Nuevas tendencias en Educación Física, Deporte y Recreación, 9,19-22

Newton, M. L., Duda J. L., y Yin, Z. (2000). Examination of the psychometric properties of the Perceived Motivational Climate in Sport Questionnaire-2 in a sample of female athletes. Journal of Sports Sciences, 18, 275-290.

Nicholls, J. G. (1984). Conceptions of ability and achievement motiva-tion. In R. Ames y C. Ames (Eds.), Research on motivation in education: Student motivation (Vol. I, pp. 39 73). New York: Academic Press.

Nicholls, J. G. (1989). The competitive ethos and democratic education. Cambridge, MA, US: Harvard University Press.

Ntoumanis, N. (2002). Motivational clusters in a sample of British physical education classes. Psychology of Sport and Exercise, 3(3), 177-194.

Ntoumanis, N. (2005). A prospective study of participation in optional school physical education using a self-determination theory framework. Journal of Educational Psychology, 97(3), 444-453.

Ntoumanis, N. y Vazou, S. (2005). Peer motivational climate in youth sport: measurement development and validation. Journal of Sport and Exercise Psychology, 27, 432-455.

Ntoumanis, N., y Biddle, S.J.H. (1999). Areview of motivational climate in physical activity. Journal of Sports Sciences, 17, 643665.

Ommundsen, Y, Roberts, G.C., Lemyre, P.N. y Miller, B.W. (2005). Peer relationships in adolescent competitive soccer : Associations to perceived motivational climate, Achievement goals and perfectionism. Journal of Sports Sciences, 23, 977-989.

Papaioannou, A. (1994). Development of a questionnaire to measure achievement goals in physical education. Research Quarterly for Exercise and Sport, 65, 11-20.

Papaioannou, A. (1995). Motivation and goal perspectives in children's physical education. In S.J.H. Biddle (Ed.), European perspectives on exercise and span psychology (pp. 245-269). Champaign, IL: Human Kinetics.

Pensgaard, A. M., Roberts, G. C. y Ursin, H. (1999). Motivational factors and coping strategies of Norwegian Paralympic and Olympic winter sport athletes. Adapted Physical Activity Quarterly, 16, 238250.
Roberts, G. C., Treasure, D. C., y Balagué, G. (1998). Achievement goals in sport: The development and validation of the Perception of Success Questionnaire. Journal of Sport Sciences, 16, 337 - 347.

Robinson, T. T., \& Carron,A. V. (1982). Personal and situational factors associated with dropping out versus maintaining participation in competitive sport. Journal of Sport Psychology, 4(4), 364-378.

Romero, S., Garrido, M.E. y Zagalaz, M.L. (2009). El comportamiento de los padres en el deporte. Retos. Nuevas tendencias en Educación Física, Deporte y Recreación, 15, 29-34.

Ruiz-Risueño, J. y De la Cruz, E. (2008). El papel del entrenador en la capacidad decisional de los jugadores. Retos. Nuevas tendencias en Educación Física, Deportey Recreación, 13, 5-10.

Ruiz, F. y García, M.E. (2003). Tiempo libre, ocio y actividad física en los adolescentes. La influencia de los padres. Retos. Nuevas tendencias en Educación Física, Deporte y Recreación, 6, 13-20.

Ryska,T.A., Hohensee, D., Cooley, D., \& Jones, C. (2002). Participation motives in predicting sport dropout among Australian youth gymnasts. North American Journal of Psychology, 4(2), 199-210.

Sousa, C., Torregrosa, M., Viladrich, C., Villamarín F., y Cruz, J. (2007). The commintment of young soccer players. Psicothema, 19, 256262.

Treasure, D. C., Duda, J. L., Hall, H. K., Roberts, G. C., y Ames, C. (2001). Clarifying misconceptions and misrepresentations in achievement goal research in sport: Aresponse to Harwood, Hardy, and Swain. Journal of Sport y Exercise Psychology, 23(4), 317-329.

Vallerand, R.J. y Thill, E.E. (1993). Introduction à la psychologie de la motivation. Montreal : Etudes Vivantes.

Van Yperen, N.W. y Duda, J.L. (1999). Goal orientations, beliefs about success, and performance improvement among young elite Dutch soccer players. Scandinavian Journal of Medicine and Science in Sport, 9, 358-364.

Vazou, S., Ntoumanis, N., y Duda, J. L. (2005). Peer motivational climate in youth sport: A qualitative inquiry. Psychology of Sport and Exercise, 6(5), 497-516.

Walling, M.D., Duda, J.L. y Crawford, T. (2002). Goal orientations, outcome, and responses to youth competition among high/low perceivedability athletes. International Journal of Sport Psychology, 37(2), 115-122.

White, S. A. (1996). Assessing the predictive validity of the parentinitiated motivational climate questionnaire to goal orientation in female volleyball players. Pediatric Exercise Science, 8, 122-129.

White, S.A. (1998). Adolescent goal profiles, perceptions of the parentinitiated motivational climate, and competitive trait anxiety. Sport Psychologist, 12(1), 16-28.

White, S. A., Kavussanu, M. y Guest, S. M. (1998). Goal orientations and perceptions of the motivational climate created by significant others. European Journal of Physical Education, 3, 212-228.

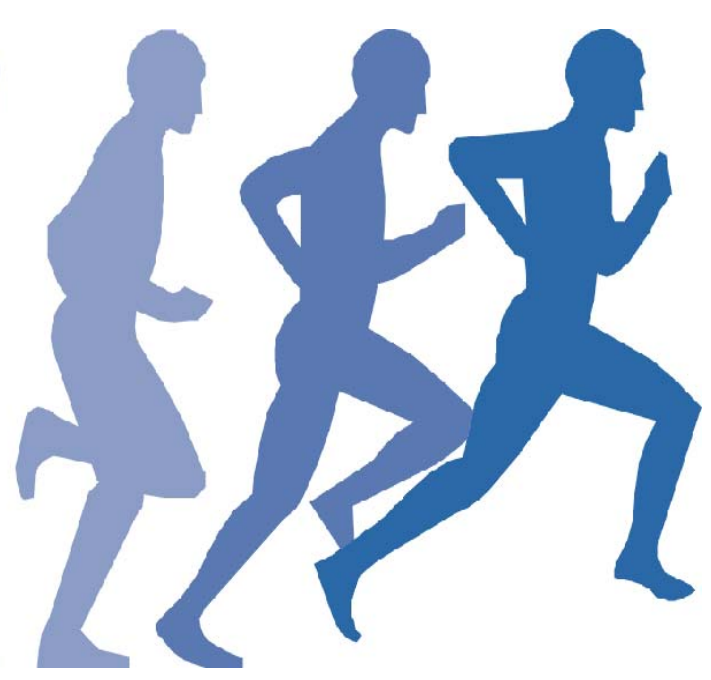

